\title{
Variability in Fluoroscopic Time during Interventional Non-Cardiac Procedures Performed Outside of the Radiology Department
}

\author{
Murdhi A. Al Harbi' ${ }^{1}$ Abdullah H. Al Malki' ${ }^{1}$, Saeed A. Al Ahmari' ${ }^{1}$, Khaled Soliman²* \\ ${ }^{1}$ Diagnostic Radiology and Medical Imaging Department, Prince Sultan Military Medical City, Riyadh, KSA \\ ${ }^{2}$ Medical Physics Department, Prince Sultan Military Medical City, Riyadh, KSA \\ Email: ^Khaledsoliman61@gmail.com
}

How to cite this paper: Al Harbi, M.A., Al Malki, A.H., Al Ahmari, S.A. and Soliman, K. (2018) Variability in Fluoroscopic Time during Interventional Non-Cardiac Procedures Performed Outside of the Radiology Department. International Journal of Medical Physics, Clinical Engineering and Radiation Oncology, 7, 464-471.

https://doi.org/10.4236/ijmpcero.2018.74039

Received: August 13, 2018

Accepted: October 8, 2018

Published: October 11, 2018

Copyright $(9) 2018$ by authors and Scientific Research Publishing Inc. This work is licensed under the Creative Commons Attribution International License (CC BY 4.0).

http://creativecommons.org/licenses/by/4.0/

\begin{abstract}
Purpose: Increasing physician awareness of patient exposure to radiation is an important step towards the reduction of potentially harmful effects of radiation. Published studies demonstrated that providing physicians with feedback regarding their fluoroscopy time leads to a reduction in average fluoroscopy times. The aim of this work was to analyze and publish our medical center data observed during the past year; fluoroscopy time (FT), Dose Area Product (DAP) and cumulative dose (CD) were monitored for radiation protection purposes. Methods: Fluoroscopy time is one of multiple radiation dose indices used in radiation safety auditing. Such auditing is nowadays turning into requirement of patient care safety and quality improvement; as indicated by accreditation bodies both nationally and internationally. All non-cardiac procedures performed outside radiology department by surgeons and interventionists are viewed. FT, DAP and CD are extracted for analysis. Results: a total of 846 cases were studied (643 orthopedic, 99 others, 73 urology, 17 chest, 7 vascular and 4 ERCP cases). Mean FT was 1.3 minutes, mean $\mathrm{CD}$ to the patient was $12.98 \mathrm{mGy}$ and the mean DAP was $4.53 \mathrm{~Gy} / \mathrm{cm}^{2}$. The longest FT noted was $55 \mathrm{~min}$. The maximum $\mathrm{CD}$ was $904 \mathrm{mGy}$ and the maximum DAP was $689 \mathrm{~Gy} / \mathrm{cm}^{2}$. Using spearman's correlation test we found out that there is a significant correlation between FT and DAP (correlation coefficient $=0.615$, p. value $<0.001)$. There is a significant correlation between FT and CD (correlation coefficient $=0.628$, p. value $<0.001$ ). Conclusion: Information about FT that used in each procedure can be used as a tool for patient dose optimization. As we found a significant correlation between $\mathrm{DAP}$ as well as $\mathrm{CD}$. Reducing fluoroscopic time (FT) is a radiation protection
\end{abstract}


goal, since it serves the purpose of protection for both the patient and the workers.

\section{Keywords}

Fluoroscopy Time, Interventional Radiology, Cumulative Dose, Dose Area Product

\section{Introduction}

Interventional procedures utilizing fluoroscopy is widely used by many medical specialties like, orthopedics, urology, gastroenterology, and vascular divisions, were C-arm fluoroscopy machine is mainly used for guidance. During these procedures the fluoroscopy is ordered by the surgeon who is the operation team leader and the radiation starts and terminated upon his request. The fluoroscopy procedures radiation doses carry risks of stochastic or deterministic injury to the patient and the highest dose is to the skin at the entrance site of radiation beam [1]. The magnitude of radiation dose received by the patients during interventional procedures is dependent on the method used by the surgeon operating the machine in the operating theater; he can reduce the fluoroscopy time resulting in reduction of the radiation dose [2] [3].

The radiation doses are given as skin doses. Fluoroscopy time has been used as a surrogate for skin dose, but it is a poor proxy because it does not account for factors such as radiation dose from radiographic or fluoroscopic images, differences in fluoroscopic dose rate, or movement of the radiation field on the patient's skin [4]. Radiation dose should be monitored throughout the procedure and recorded after the procedure [5]. This study is targeted to assess all fluoroscopic procedures performed outside radiology department considering the issue that non-radiologist physicians don't have an adequate radiation safety program in their training fellowship beside the lack of guidelines that provides a dose reference levels for many of the fluoroscopic procedures performed in theatre rooms. In order to assist physicians to request radiological studies weighting the risk in relation to the benefits, it is necessary for them to have the knowledge on ionizing radiation and its risks. If that knowledge is inappropriate, patients may be investigated more times and by methods that rely on higher than necessary radiation doses [6].

For the purpose of safety and optimization of radiation dose to both the patient and the workers all fluoroscopy procedures performed outside radiology shall be monitored to ensure that the radiation dose to the patient is "ALARA". In this study, fluoroscopy time and its relation with the cumulated dose during non-cardiac interventional procedures will be studied, the results will be compared with internationally reported data and the reliability of using fluoroscopy time as a surrogate for radiation exposure will be discussed. 


\section{Methods}

The radiation safety officer in diagnostic radiology monitored the fluoroscopy time registered for all procedures and the fluoroscopy time (FT) is obtained from PACS system is used as optimization and safety tool by notifying the operating physicians about their procedures radiation duration (FT) which will have an impact on their future cases.

Data of (FT) fluoroscopy time, (DAP) dose area product and cumulative dose are collected from all cases (see Figure 1) done in 2017 and analyzed. The data were presented as a feedback to the concerned physician's department and to the hospital's radiation safety committee. The data are from a tertiary care medical centre with bed capacity of more than 1600 beds. Research centre ethical committee approval was obtained before the start of this project.

Descriptive statistics was performed on the FT distribution. The potential relationship between the Fluoroscopy Time, Cumulated Dose and Dose Area Product was tested for correlation by calculating both spearman and Pearson correlation coefficients.

\section{Results}

\subsection{Relationships between Fluoroscopy Time and Cumulative Dose}

The total number of cases presented in this work is 846 cases performed from January 2017 to December 2017 in tertiary care medical Centre; histogram in Figure 1 is show the types and number of procedures performed. Table 1 below shows the data for CD, DAP and FT.

Since the distribution of fluoroscopy time, cumulative dose and dose area product does not follow a normal pattern as described below in Figures 2-4; non parametric statistics were used to perform the analysis such as $\rho$ spearman correlation coefficient in testing the potential correlation between fluoroscopy time and cumulative dose. The scatter map for Fluoroscopy Time in [min] is presented in Figure 5.

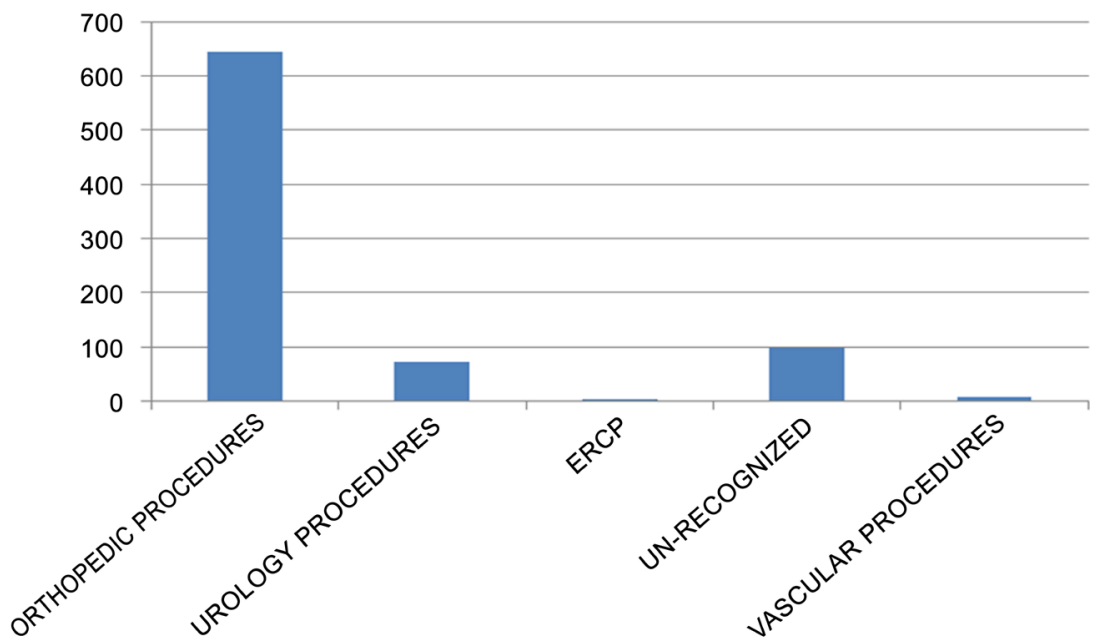

Figure 1. Data distribution per group of clinical procedures. 
Table 1. Descriptive table.

\begin{tabular}{cccccc}
\hline Variable & Min & Max & Median & mean & SD \\
\hline Cumulative Dose $(\mathrm{mGy})$ & 0 & 904 & 1.6 & 12.98 & 47.67 \\
DAP $\left(\mathrm{Gy} \cdot \mathrm{cm}^{2}\right)$ & 0 & 689 & 0.41 & 4.53 & 30.62 \\
Total F. time (in seconds) & 0 & 3300 & 27 & 78.9 & 202.18 \\
\hline
\end{tabular}

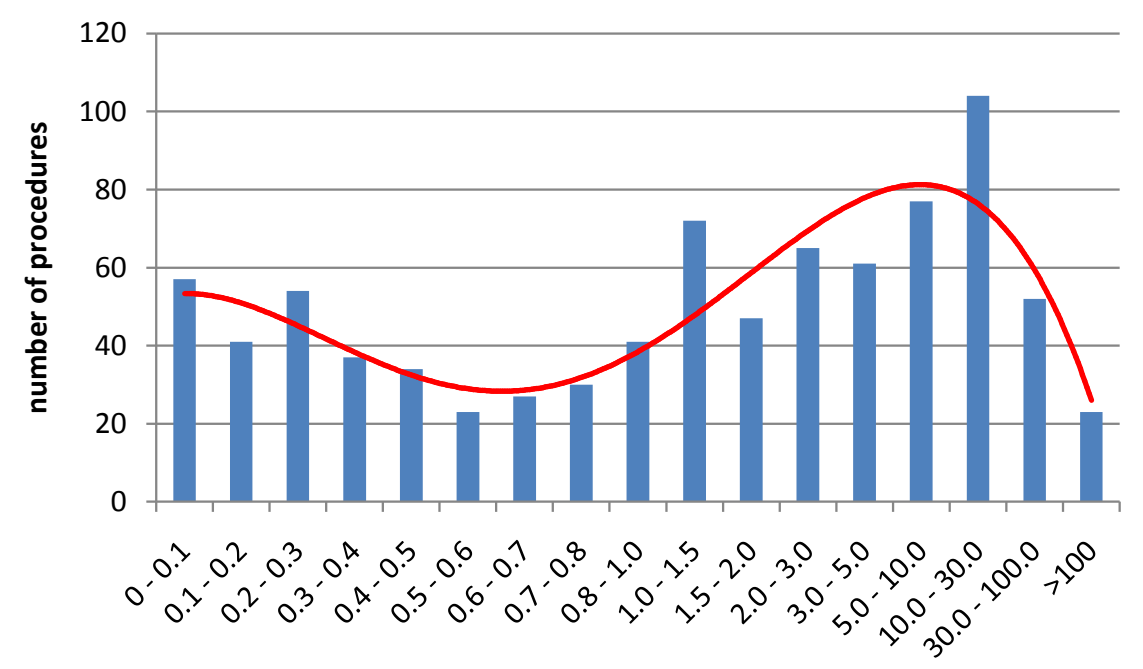

Cumulative Dose $[\mu \mathrm{Gy}]$

Figure 2. Cumulative Dose (CD) distribution.

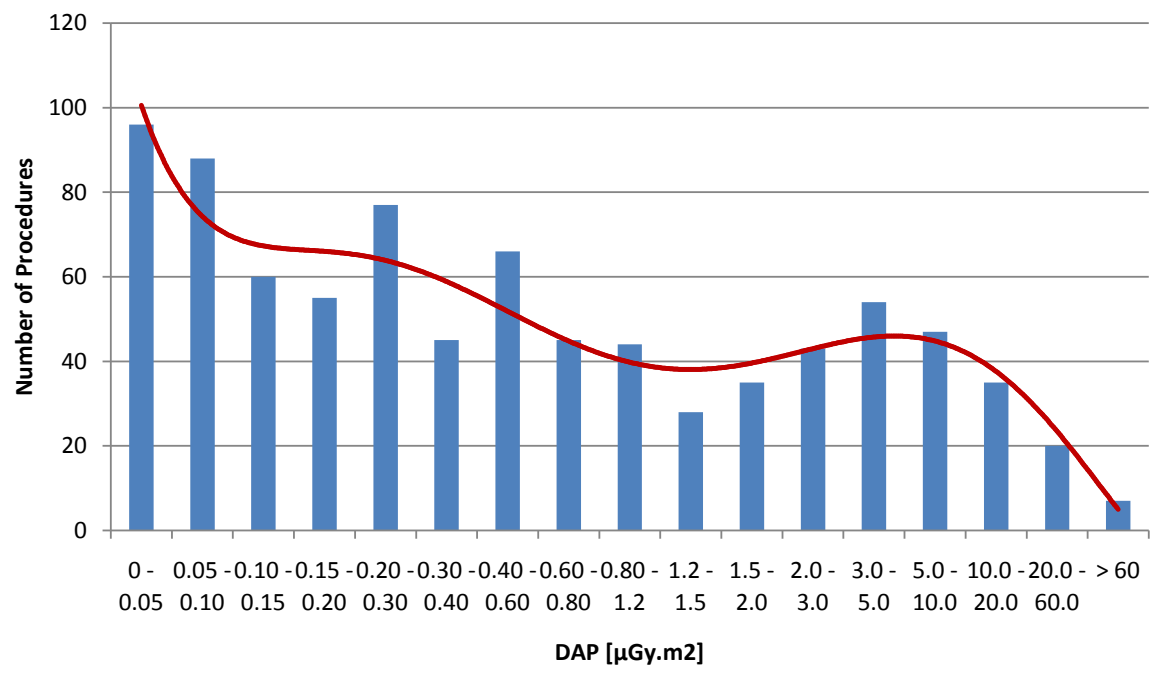

Figure 3. Dose Area Product (DAP) distribution.

There was a significant correlation between fluoroscopy time and DAP ( $\rho$ spearman correlation coefficient $=0.615, \mathrm{p}$. value $<0.001$ ) There is a significant correlation between fluoroscopy time and cumulative dose ( $\rho$ spearman correlation coefficient $=0.628$, p. value $<0.001$ )

The fluoroscopy times are then classified as per medical divisions performing fluoroscopic guided procedures, the majority (76\%) were orthopedic procedures. 


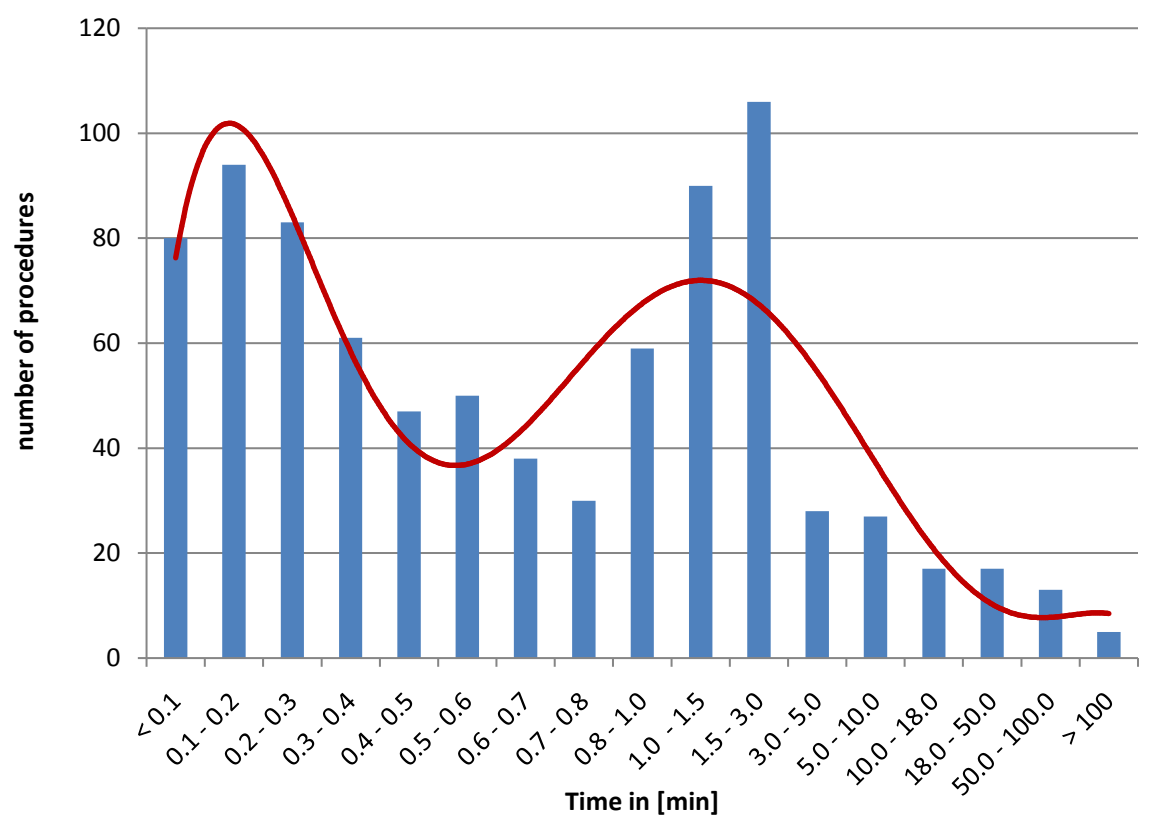

Figure 4. Distribution of fluoroscopy time (FT) for all the observed procedures.

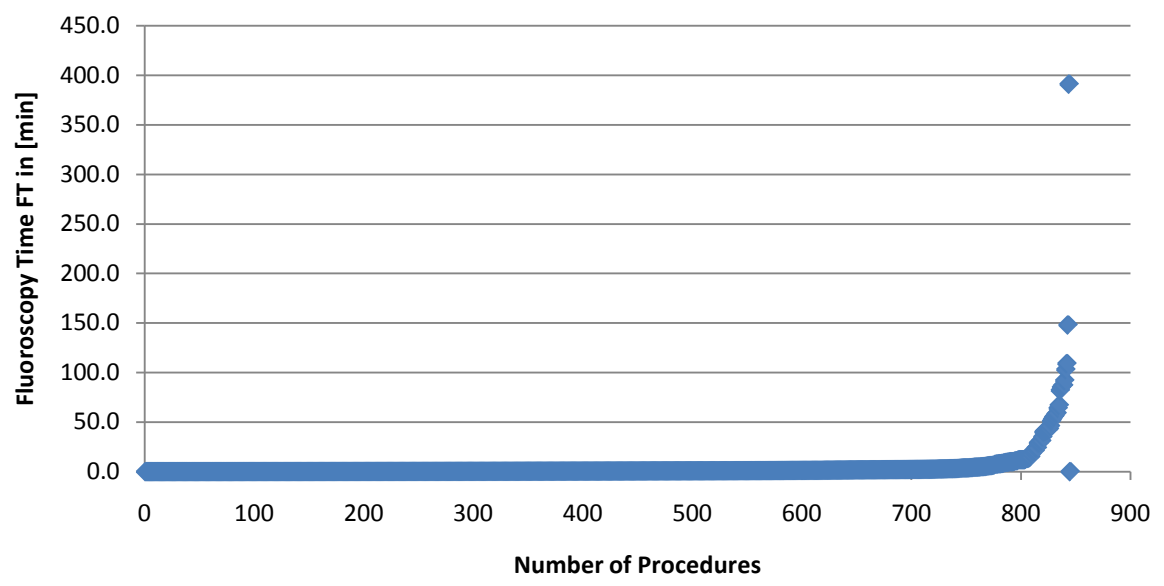

Figure 5. Scatter map for Fluoroscopy Time in [min].

The fluoroscopy time is categorized into groups as in the Figure 4.

\subsection{Fluoroscopy Time during Orthopedic Procedures}

As orthopedic procedures represent $75 \%$ of total cases of this study, we extracted them and compared our results with three published studies in Table 2.

\section{Discussion}

All fluoroscopy machines are set to notify the operator about the fluoroscopy time as it beeps at every 5 minutes of screening time, on the other hand the machines do not provide any notification at any level of dap or cumulative dose.

It important to physicians to be notified about radiation exposure to their patient during the procedure not later on by looking at the estimated entrance skin 
Table 2. Average FT and other reported studies.

\begin{tabular}{ccc}
\hline Author & $\mathbf{n}$ & $\mathbf{t}$ (minutes) \\
\hline This study & 643 & 5.06 \\
V. Tasapaki [7] & 205 & 2.2 \\
Malek [8] & 670 & 2.5 \\
Tsalafoutas [9] & 204 & 3.0 \\
\hline
\end{tabular}

dose or DAP, and this could be achieved by monitoring the fluoroscopy time while operating on the patient in the absence of DAP or CD alarms. Koichi Chida [10] reported that it is recommended to that the physicians record the fluoroscopic time when DAP cannot be monitored for estimating the maximum patient skin dose in RFCA procedures.

Yet there are many published studies confirming that fluoroscopy time is a weak dose indicator and should not be used alone to estimate the radiation dose. While we found that there is significant correlation between FT and DAP as well as $\mathrm{CD}$ and this finding could be attributed to the large number of cases included in this study 846 while other studies were reporting at most 145 cases.

Most studies that address fluoroscopy time are coned usually to a certain procedure or a specific age, especially pediatrics and this can be explained that pediatrics are of a special concern when it comes to radiation safety as their cells are less differentiated and more sensitive to radiation beside that they have a longer life expectancy [11]. However, studying the fluoroscopy time in a limited range makes the results less consistent with the findings.

Skripochnik, published an article on March 2017 about fluoroscopy time and they concluded that Fluoroscopy time shows minimal correlation with radiation delivered and therefore is a poor surrogate for radiation exposure during fluoroscopy procedures [12]. They conducted their study specifically on 145 Lower extremity endovascular intervention cases. J. McBride explored the factors that may affect the radiation dose and fluoroscopy time in RAS "renal artery stent placement procedure" [13]. The study outlined only one factor that increase patient radiation dose and fluoroscopy time which is the number of stents placed. The study was performed on 136 patients who have RAS which makes this study and its finding homogenous compared to the variable fluoroscopic procedures. Bucci et al., outlined the factors that affect patient dose and proposed some changes that may be changed to the fluoroscopy machines settings in order to minimize the patient's dose [14]. Brady, reviewed the pediatric dose from MCUG "micturition cysto-urethrogarm". The study showed that there is no correlation between physician's experience and radiation dose resulting from their selected study. The study used DAP results to assess the patient dose only [15].

In conclusion, to the best of our knowledge there was no published study covering quit large population size that addresses the variability in fluoroscopy time during procedures that utilize fluoroscopy guidance. 
We recommend an annual review of fluoroscopy time in large medical centers and the utilization of the obtained information as an optimization tool for patient and staff safety in collaboration with the medical practitioners conducting the procedures.

\section{Conclusion}

Published studies showed that fluoroscopy time (FT) is a weak dose indicator and cannot be used alone to estimate the radiation dose to the patient as it does not correlate with DAP and CD, in our study we found out throughout the 846 cases that there is a significant correlation between fluoroscopy time DAP and $\mathrm{CD}$ as well. We suggest that informing physicians about the fluoroscopy time measured during the procedures they have performed is a reliable patient dose optimization method; allowing clinician to optimize their practice.

\section{Conflicts of Interest}

The authors declare no conflicts of interest regarding the publication of this paper.

\section{References}

[1] Kim, S., Shin, J.H., Lee, J.W., Kang, H.S., Lee, G.Y. and Ahn, J.M. (2016) Factors Affecting Radiation Exposure during Lumbar Epidural Steroid Injection: A Prospective Study in 759 Patients. Korean Journal of Radiology, 17, 405-412. https://doi.org/10.3348/kjr.2016.17.3.405

[2] Choi, M.H., Choi, B.G., Jung, S.E. and Byun, J.Y. (2016) Factors Related to Radiation Exposure during Lumbar Spine Intervention. Journal of Korean Medical Science, 31, S55-S58. https://doi.org/10.18284/jss.2016.12.35.2.5

[3] Miller, D.L., Balter, S., Schueler, B.A., Wagner, L.K., Strauss, K.J. and Vañó, E. (2010) Clinical Radiation Management for Fluoroscopically Guided Interventional Procedures. Radiology, 257, 321-332. https://doi.org/10.1148/radiol.10091269

[4] Balter, S., Hopewell, J.W., Miller, D.L., Wagner, L.K. and Zelefsky, M.J. (2010) Fluoroscopically Guided Interventional Procedures: A Review of Radiation Effects on Patients' Skin and Hair. Radiology, 254, 326-341. https://doi.org/10.1148/radiol.2542082312

[5] Lee, G., Hunter, R.J., Lovell, M.J., Finlay, M., Ullah, W., Baker, V., Dhinoja, M.B., Sporton, S., Earley, M.J. and Schilling, R.J. (2015) Use of a Contact Force-Sensing Ablation Catheter with Advanced Catheter Location Significantly Reduces Fluoroscopy Time and Radiation Dose in Catheter Ablation of Atrial Fibrillation. Ep Europace, 18, 211-218. https://doi.org/10.1093/europace/euv186

[6] Madrigano, R.R., Abrão, K.C., Puchnick, A. and Regacini, R. (2014) Evaluation of Non-Radiologist Physicians' Knowledge on Aspects Related to Ionizing Radiation in Imaging. Radiologiabrasileira, 47, 210-216. https://doi.org/10.1590/0100-3984.2013.1840

[7] Tsapaki, V., Tsalafoutas, I.A., Fagkrezos, D., Lazaretos, I., Nikolaou, V.S. and Efstathopoulos, N. (2015) Patient Radiation Doses in Various Fluoroscopically Guided Orthopaedic Procedures. Radiation Protection Dosimetry, 168, 72-75. https://doi.org/10.1093/rpd/ncv007 
[8] Malek, S., Davies, E., Malek, I.A., Rawal, A., Singh, A. and Harvey, R.A. (2007) Trauma Surgery and Risk of Radiation Injury to Patients. European Journal of Orthopaedic Surgery \& Traumatology, 17, 23-28. https://doi.org/10.1007/s00590-006-0127-7

[9] Tsalafoutas, I.A., Tsapaki, V., Kaliakmanis, A., Pneumaticos, S., Tsoronis, F., Koulentianos, E.D. and Papachristou, G. (2007). Estimation of Radiation Doses to Patients and Surgeons from Various Fluoroscopically Guided Orthopaedic Surgeries. Radiation Protection Dosimetry, 128, 112-119. https://doi.org/10.1093/rpd/ncm234

[10] Chida, K., Saito, H., Otani, H., Kohzuki, M., Takahashi, S., Yamada, S., Shirato, K. and Zuguchi, M. (2006) Relationship between Fluoroscopic Time, Dose-Area Product, Body Weight, and Maximumradiation Skin Dose in Cardiac Interventional Procedures. American Journal of Roentgenology, 186, 774-778. https://doi.org/10.2214/AJR.04.1653

[11] Shope, T.B. (1996) Radiation-Induced Skin Injuries from Fluoroscopy. Radiographics, 16, 1195-1199. https://doi.org/10.1148/radiographics.16.5.8888398

[12] Skripochnik, E. and Loh, S.A. (2017) Fluoroscopy Time Is Not Accurate as a Surrogate for Radiation Exposure. Vascular, 25, 466-471. https://doi.org/10.1177/1708538117698342

[13] McBride, J., Schueler, B., Oderich, G. and Misra, S. (2013) An Analysis of the Factors Influencing Radiation Dose and Fluoroscopic Time during Renal Stent Placement. Vascular and Endovascular Surgery, 47, 462-466. https://doi.org/10.1177/1538574413495460

[14] Bucci, R.V., Gaisie, G., Cheryl Miller, R.T., Rubin, M. and Springer, A. (2014) Radiation Dose Reduction in Fluoroscopy. Radiology Management, 9-10.

[15] Brady, Z. (2016) Radiation Dose in Fluoroscopy: Experience Does Matter. Journal of Medical Imaging and Radiation Oncology, 60, 457-458. https://doi.org/10.1111/1754-9485.12485 